\title{
Volumetric Modulated Arc Therapy followed by bilateral mastectomy for treating synchronous cancer and sarcoma - a rare case report
}

\author{
Jacek Gałecki ${ }^{1}$, Klaudia Bęczkowska ${ }^{1}$, Piotr Mężeński ${ }^{2}$
}

Purpose. Bilateral postmastectomy irradiation (BPMI) poses a challenge to radiotherapists. The difficulty lies whenever targets are large, irregular and lying at different depths. When breathing movements occur it is very hard to obtain homogenous dosing on such targets and also to not exceed those doses acceptable for at-risk organs, particularly the lungs and heart. The presented study is an example of using a single isocenter Volumetric Modulated Arc Therapy (VMAT) technique for a BPMI case.

Materials and methods. We report the case of a 66-year-old woman with simultaneous malignant sarcoma of the right breast and an invasive ductal carcinoma of the left breast. After modified radical mastectomy of the left breast and simple mastectomy of the right breast, the patient underwent adjuvant irradiation of the bilateral chest wall and left axillary and supraclavicular nodes region. Planning Target Volumes (PTV) and organs at risk (OAR) were delineated on CT scans. A single isocenter VMAT plan was created. The radiotherapy consisted of 50 Gy delivered in $2 \mathrm{~Gy}$ per fraction to the left side and 56.5 Gy delivered in 2.25 Gy per fraction to the right side in 25 fractions, five times a week over 5 weeks.

Results. The left and right CTV (Clinical Target Volume) received $90 \%$ of the prescribed dose in $100 \%$ of the CTV volume. The mean heart dose was $14.4 \mathrm{~Gy}$ and V25 for the heart was $8.9 \%$. The mean lung dose was $16.6 \mathrm{~Gy}$ and the V20 for both lungs was $28.3 \%$. The dose to the anterior wall area of the heart was exceeded according to established constraints, but was accepted. One session of irradiation lasted about four minutes during which the patient received 595 monitor units. The treatment was carried out without interruption and complications. The early and late skin reaction was assessed as being second grade. After one year of follow-up, the patient did not present any serious side effects or recurrence.

Conclusions. Synchronous irradiation of both sides of the chest wall after bilateral mastectomy with a single isocenter VMAT technique is possible and well tolerated.

NOWOTWORY J Oncol 2017; 67, 1:48-53

Key words: volumetric modulated arc therapy, bilateral postmastectomy irradiation, synchronous breast malignancy

\section{Introduction}

The clinical need for performing bilateral postmastectomy chest wall irradiation occurs rarely because of the low incidence rate of synchronous bilateral breast cancer (BBC), at about 2\% [1,2]. Breast sarcoma (BS) is an even much rarer malignancy [3], thus the presented case of both these can- cers appearing together is rather an extreme coincidence. Such situations require nonconventional management using modern techniques of adjuvant radiotherapy. This study presents the use of one such technique; a single isocentre Volumetric Modulated Arc Therapy (VMAT) for bilateral synchronous postmastectomy irradiation.

\footnotetext{
${ }^{1}$ Department of Radiotherapy I 


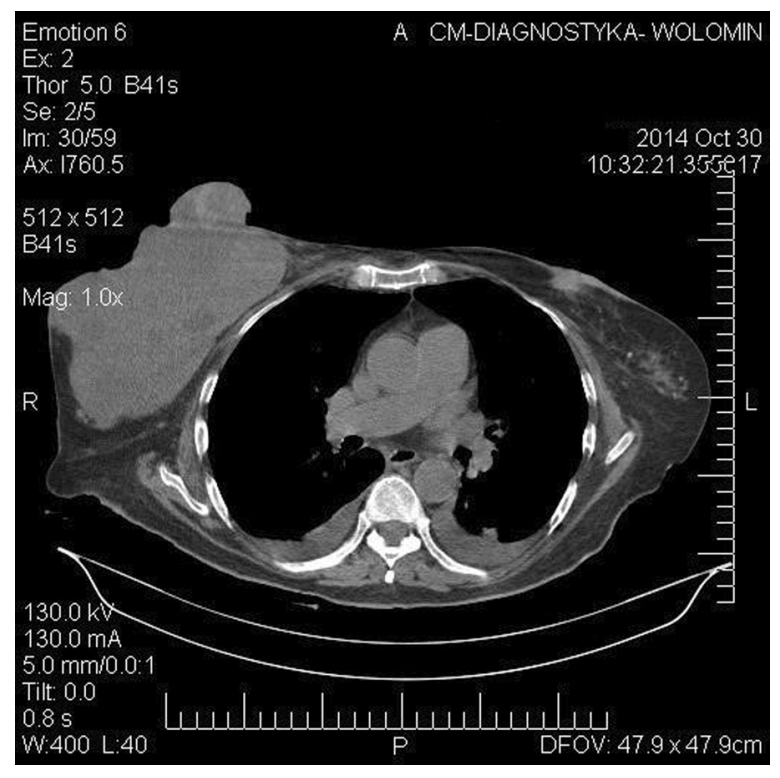

Figure 1. Computed tomography before surgery

\section{Case report \\ Patient's history and diagnoses}

In November 2014, a 66-year old woman with type 2 diabetes and arterial hypertension was admitted to emergency care, suffering severe anaemia due to blood loss from a huge ulcerous right breast tumour. The patient had previously noticed a lump in her right breast about 1.5 years ago that had not been subject to diagnoses nor treatment, but this had rapidly grown within the last month. A family history of breast cancer was not reported. Breast palpation presented $15 \mathrm{~cm}$ bleeding and an ulcerous mass occupying the entire right breast which was mobile in relation to the pectoralis major muscle. No obvious lumps were found in the right axilla. The same examination revealed a palpable $3.5 \mathrm{~cm}$ tumour in the lateral quadrants of the left breast. A palpable and movable $1.5 \mathrm{~cm}$ nodule was also found in the left axillary fossa. The computed tomography (CT) of the thorax confirmed a $17.5 \times 10.3 \mathrm{~cm}$ polycyclic tumour in the right breast and a tumour on the border between the external quadrants of the left breast of $3.0 \times 1.2 \mathrm{~cm}$ size and a $1.3 \mathrm{~cm}$ lymph node in the left axilla (Fig. 1).

The abdominal ultrasound and brain CT showed no obviously significant aberrations in other organs. Excisional biopsy of the right breast tumour demonstrated a malignant solitary fibrous tumour. Ultrasound-guided percutaneous biopsy of the left breast lesion showed an invasive ductal carcinoma. Based on histopathological diagnosis and clinical stage, the patient was qualified for surgery of both breast lesions at the same time. Modified radical mastectomy of the left breast and simple mastectomy of the right breast was thereby performed. The final pathological examination showed in the left breast: - an invasive ductal carcinoma NST, a histological grade 2 (G2) rating, pT2 $(3.0 \mathrm{~cm}$ in its greatest dimension) and $\mathrm{pN} 3 \mathrm{c}$ (four nodes involved out of twelve excised) - oestrogen receptor-positive, progesterone receptor - negative, HER2 - negative and Ki67 - 10\%. In the right breast, the diagnosis of a malignant solitary fibrous tumour was confirmed (at its greatest dimension of $21.0 \mathrm{~cm}$ ). Surgical margins were free of malignancy.

Because of concomitant diseases and established ischemic heart disease, as shown by echocardiography, the patient was not qualified for adjuvant chemotherapy but was given adjuvant radiotherapy and hormone-therapy with Tamoxifen. Due to the high risk of the loco-regional tumours recurring, the patient was synchronously irradiated on both sides of the chest wall and on the left axillary and supraclavicular lymph nodes region. The radiotherapy consisted of 50 Gy delivered in 2 Gy per fraction to the left side and $56.5 \mathrm{~Gy}$ delivered in $2.25 \mathrm{~Gy}$ per fraction to the right side, five times a week over 5 weeks. The early and late skin reaction was assessed by the RTOG scale $[4,5]$.

\section{Imaging and treatment planning}

CT imaging was performed on the treated patient in a supine position with both arms above the head. During the CT scans and in subsequent radiation therapy, a $1 \mathrm{~cm}$ tissue equivalent bolus was added around the patient chest wall. On the left side, an additional wax bolus was added to obtain a homogenous dose distribution on the irregular shaped chest wall (Fig. 2). Using CT cross sections, the Clinical Target Volume (CTV) for both sides were delineated. The right side CTV included only the chest wall, whilst the left side CTV included the chest wall and supraclavicular and axillary lymph nodes. Planning Target Volume (PTV) was established by adding an isotropic margin of $0.6 \mathrm{~cm}$ to the CTV (Fig. 3). The organs at risk (OAR) defined by CT were: both lungs, heart, coronary arteries and spinal cord. Our own protocol for delineating CTV and OAR dose were based on the Danish Breast Cancer Cooperative Group, QUANTEC and ESTRO consensus guidelines [6-11]. The mean dose to the heart should be below 26 Gy and V25 volume below $10 \%$. In the lungs, the V20 volume should be below $30 \%$ and the mean lung dose less than $20 \mathrm{~Gy}$, whilst the maximum dose to spinal cord should be below $50 \mathrm{~Gy}$. Coronary arteries were schematically delineated as an area of the anterior wall of the heart (Fig. 3) [12]. A single isocentric VMAT plan was prepared by a medical physicist using the Eclipse treatment planning system (version 10). The treatment plan was based on two $6 \mathrm{MV}$ photon arcs (clockwise and anti-clockwise). Each arc was divided into upper and lower subfields (Fig. 3). The Analytical Anisotropic Algorithm dose calculation algorithm was used. A simultaneous optimization for both sides with different doses per fraction was performed. 


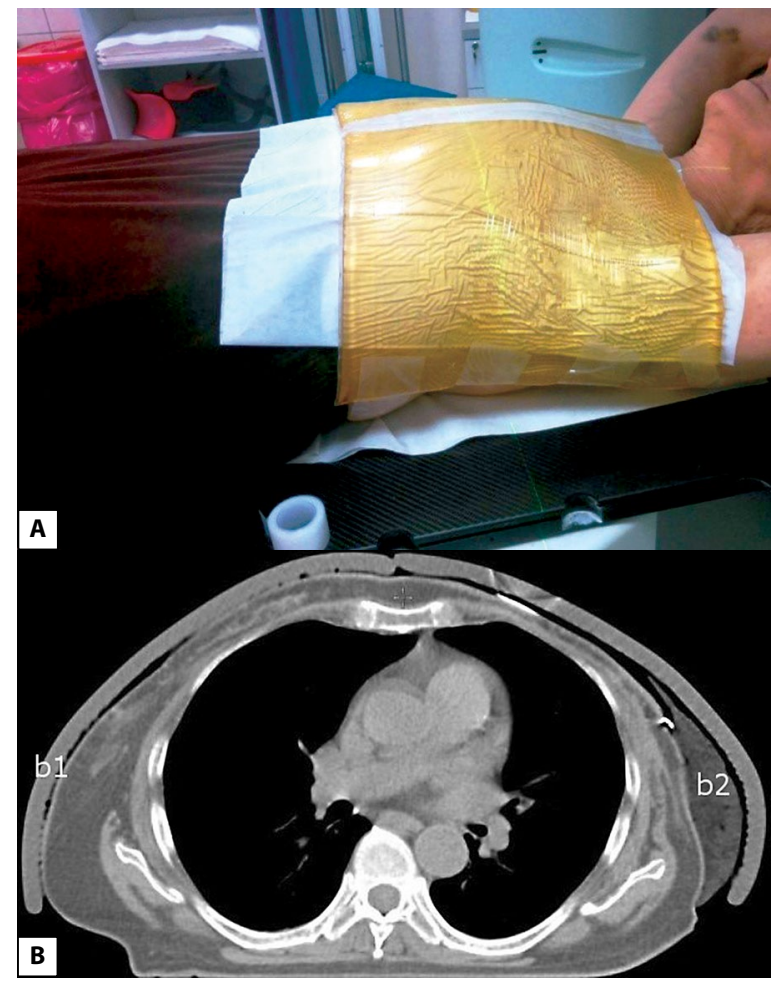

Figure 2. The patient in a supine position with a $1 \mathrm{~cm}$ bolus covering the chest wall (A); CT cross section with a $1 \mathrm{~cm}$ bolus (b1) and additional bolus (b2) on the left side (B)

\section{Quality Assurance (QA) of the treatment plan}

This was performed on the Varian EPID (Electronic Portal Imaging Devices). The differences between calculated and measured dose distributions were evaluated by the gamma parameter [13].

\section{Treatment delivery with image-guidance}

The patient was treated with the TrueBeam linac accelerator. The daily patient setup was verified by planar setup fields and Cone Beam Computed Tomography (CBCT).

\section{Results}

For the target volumes CTV and PTV the following dose distribution parameters are presented.

The Left CTV $90 \%$ and $95 \%$ of the prescribed dose cover $100 \%$ and $98.9 \%$ of the structure with a standard deviation of dose (STD) values at $2.4 \%$. For left PTV $90 \%$ and $95 \%$ of the prescribed dose cover $99.3 \%$ and $94 \%$ volume of the structure, STD $2.8 \%$. For right CTV $90 \%$ and $95 \%$ of the prescribed dose cover $100 \%$ and $98.2 \%$ of the structure volume and cover $97.6 \%$ and $90.7 \%$ of the PTV volume. The STD values are $2.4 \%$ for the CTV and $3.4 \%$ for the PTV (Figs. 4-6). The mean heart dose was $14.4 \mathrm{~Gy}$ and theV25 for heart was $8.9 \%$. The mean lungs dose was $16.6 \mathrm{~Gy}$, and theV20 for both lungs was $28.3 \%$. The maximum dose to the spinal cord was $21 \mathrm{~Gy}$. The additional organ at risk "the coronary

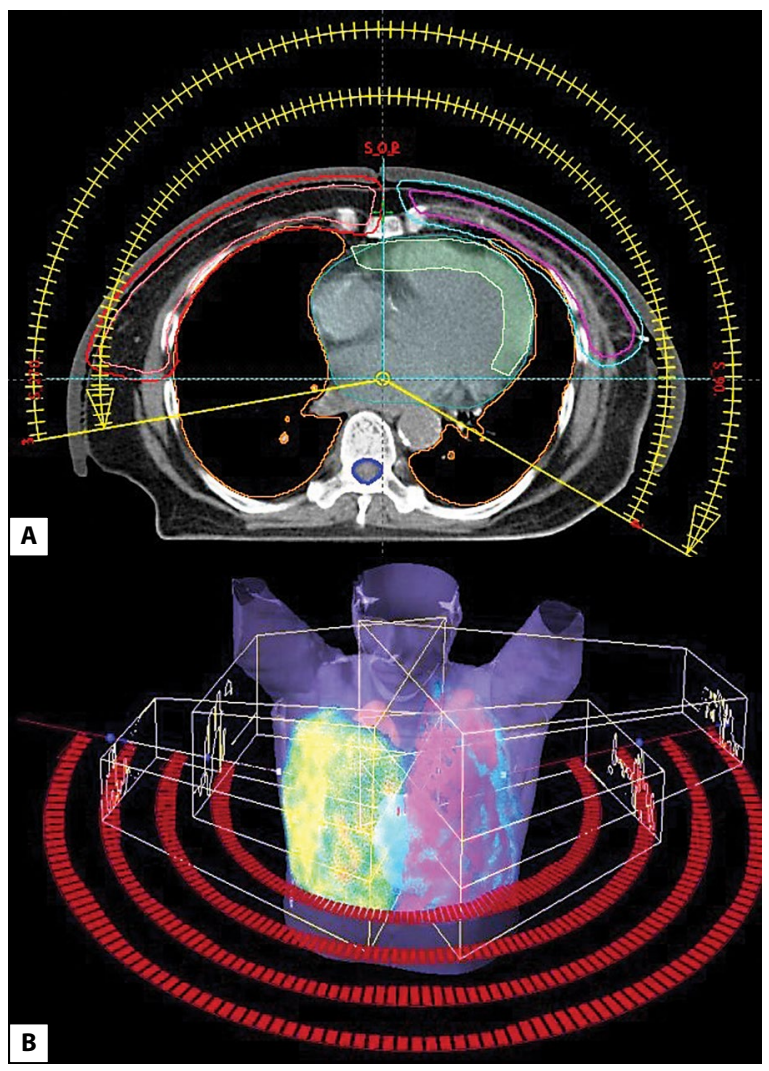

Figure 3. Therapeutic arc arrangements and contours of the target volumes and OAR. Pink-right CTV, red-right PTV, magenta-left CTV, cyan-left PTV. Orange-lung's, dark green-heart, light green-coronary arteries, blue-spinal cord (A); View of therapeutic fields (B)

arteries" was involved in a optimisation process to reduce the dose to the heart. In the coronary arteries structure, the volume of $20 \mathrm{~Gy}$ was minimized. In the plan to pass the QA verification, the gamma parameter $(3 \%, 3 \mathrm{~mm})$ value was 96.5\% (tolerance 95\%). Unfortunately the dose to the heart's anterior wall area was exceeded, according to established constraints, but was accepted (Fig. 7; Tab. I). One session of irradiation lasted about four minutes during which the patient received 595 monitor units. The treatment was carried out without interruptions and complications. The early and late skin reaction was assessed as being second grade (Fig. 8). After one year of follow-up, the patient did not present any serious side effects or recurrence.

\section{Discussion}

Postmastectomy bilateral irradiation (PMBI) constitutes a challenge for the radiotherapist. Due to large and irregular targets lying at different depths, in conjunction with breathing movements, it is very difficult to achieve properly homogenous dosing onto targets and not to exceed acceptable doses for OAR; particularly the lungs and heart. Nowadays, the most frequently used postmastectomy irradiation techniques are Mono Isocentric Conformal 


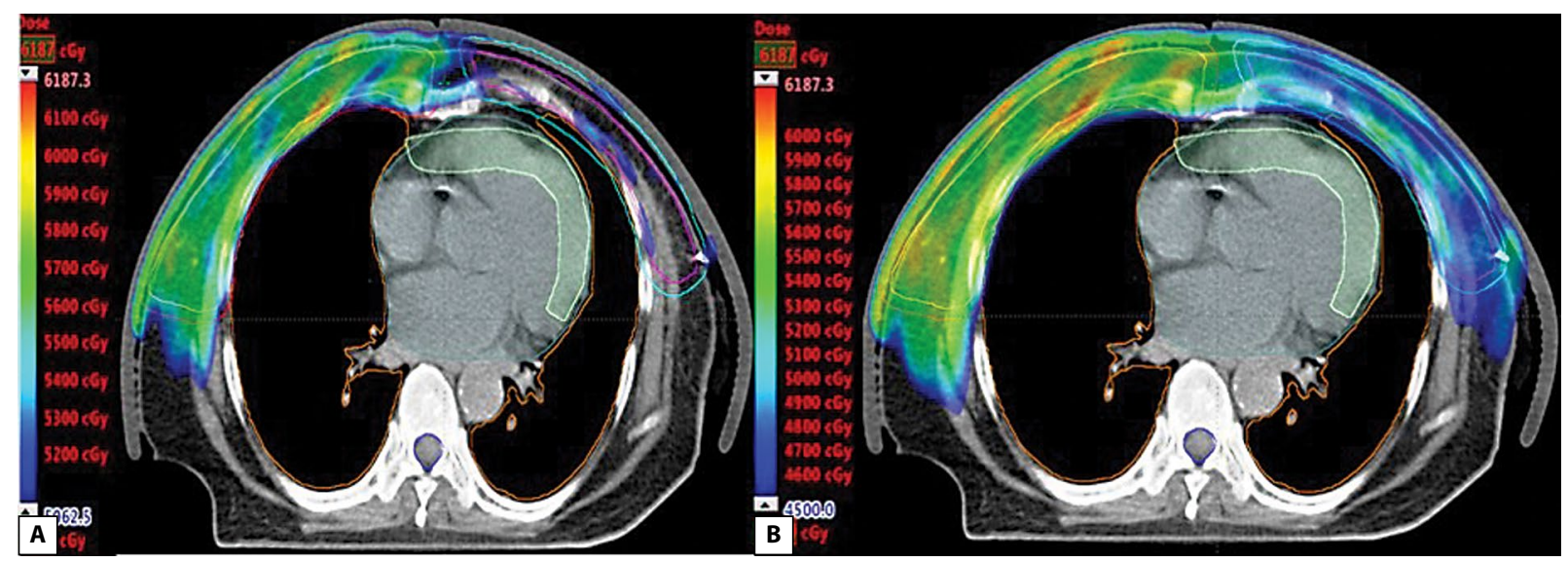

Figure 4. Dose distribution. A. Right side PTV isodose level from $90 \%$ of prescribed dose (5062,5 cGy). B. Left side PTV isodose level from $90 \%$ of prescribed dose (4500 cGy)

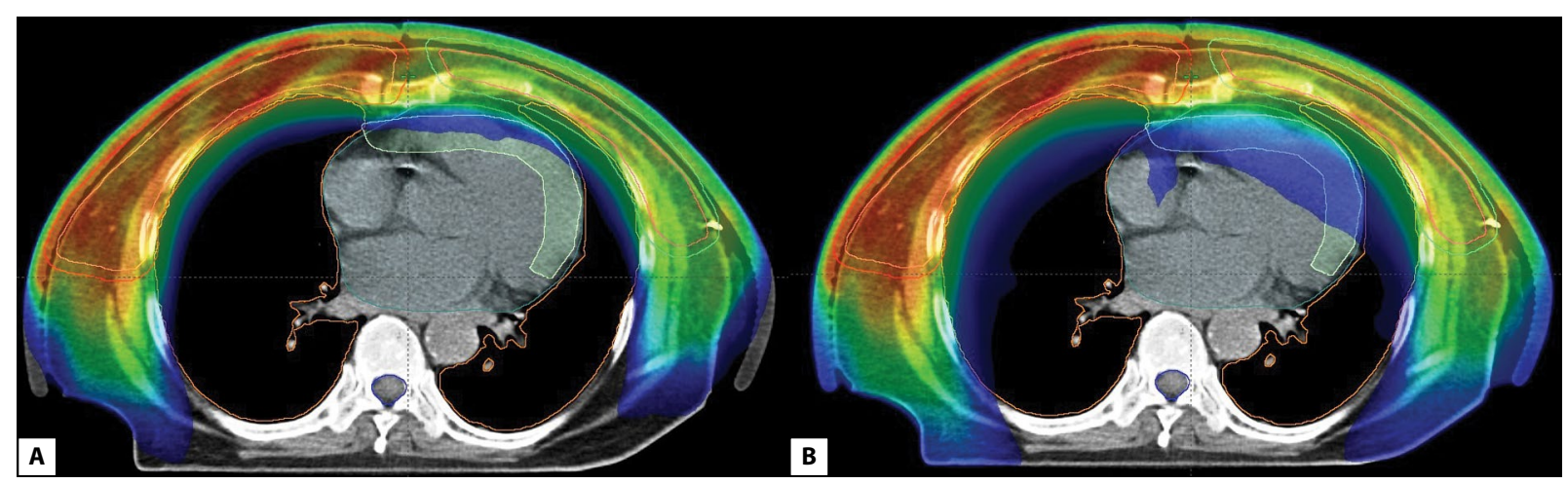

Figure 5. Dose distribution of: A. 20 Gy and more B. 10 Gy and more

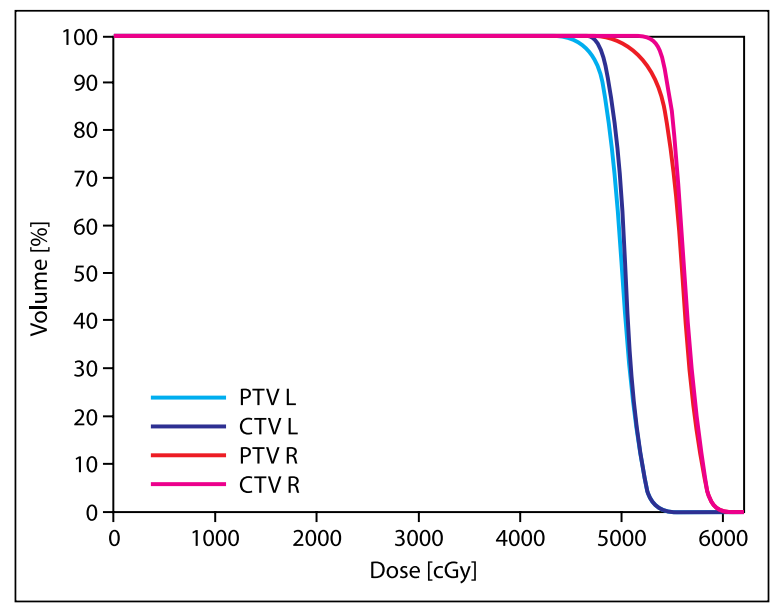

Figure 6. Dose Volume Histogram of the CTV (Clinical Target Volume) and PTV (Planning Target Volume) in the presented irradiated patient. CTV L and PTV L — left side target volumes; CTV R and PTV R — right side target volumes

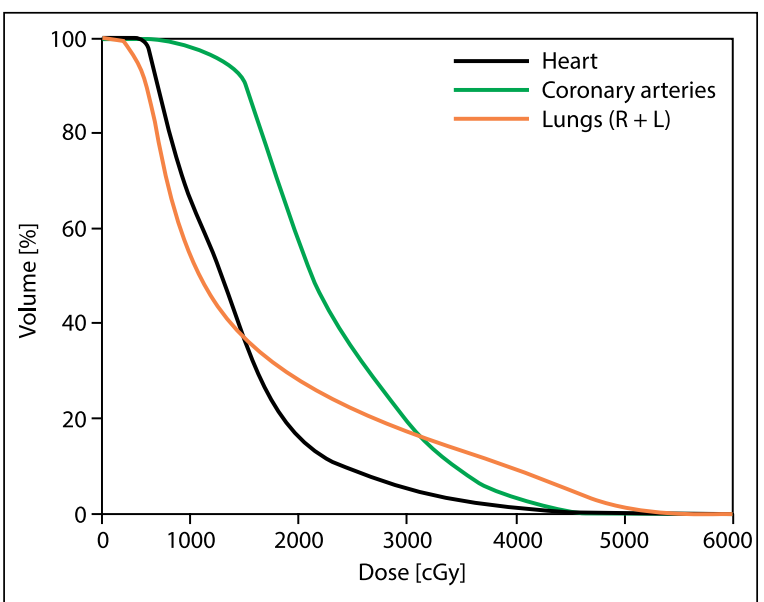

Figure 7. Dose Volume Histogram of the organs at risk (OAR) in the presented irradiated patient 
Table I. Doses in the organs at risk

\begin{tabular}{lcc}
\hline Organ at risk & $\begin{array}{c}\text { Doses achieved in } \\
\text { presented case }\end{array}$ & $\begin{array}{c}\text { Established doses } \\
\text { constraints }\end{array}$ \\
\hline Heart & $\mathrm{V}_{25}=8.9 \%$ & $\mathrm{~V}_{25}<10 \%$ \\
$\mathrm{D}_{\text {mean }}=14.4 \mathrm{~Gy}$ & $\mathrm{D}_{\text {mean }}<26 \mathrm{~Gy}$ \\
Lungs & $\mathrm{D}_{\text {mean (desirable) }}<10 \mathrm{~Gy}$ \\
& $\mathrm{D}_{\text {mean }}=16.6 \mathrm{~Gy}$ & $\mathrm{D}_{20}<30 \%$ \\
Coronary artery & $\mathrm{D}_{20}=57.5 \%$ & $\mathrm{~V}_{\text {mean }}<20 \mathrm{~Gy}$ \\
& $\mathrm{D}_{\text {mean }}=23.8 \mathrm{~Gy}$ & $\mathrm{D}_{\text {mean }}<17 \mathrm{~Gy}$
\end{tabular}

Radiotherapy, Intensity Modulated Radiation Therapy (IMRT) or Volumetric Modulated Arc Therapy (VMAT) [14]. Due to the extensive area that requires radiation treatment use of single-isocenter IMRT is not possible due to the limitations of maximum size of the therapeutic field. Nevertheless, the use of IMRT plans based on two isocenters result in a summation of the dose in the sternum region to $75 \mathrm{~Gy}$. That is why, VMAT technique with single-isocenter was used. Besides, using conventional IMRT techniques, the irradiation time is prolonged due to two separate isocentres used. In our case, one session of irradiation lasted only four minutes. VMAT delivers the same radiation doses faster with less monitor units than for conventional IMRT, as well as enabling organs at risk to be often better shielded/ /spared [15]. However, due care and attention are required for IMRT or VMAT techniques, which are only used for specific clinical circumstances because of the larger areas of normal tissues exposed to small doses compared to conformal methods. The presented patient underwent a left radical mastectomy because of cancer, but only a simple mastectomy on the right side due to sarcoma was performed because this type of neoplasm spreads mostly through blood vessels and does not require axillary dissection. For such reasons the left side chest wall and supraclavicular and axillary region were together exposed to irradiation, but only the right side chest wall received such irradiation. These two sides of treatment with a different diagnosis and high risk of recurrence, required fast irradiation treatment because of concomitance diseases and so the patient was not qualified for adjuvant chemotherapy. The modern technique of VMAT irradiation was chosen so as to permit the doses to be differentiated according to the diagnosis and where both sides could be simultaneously treated. On the left and right sides, total doses was respectively given of $50 \mathrm{~Gy}$ and 56.25 Gy over 5 weeks (25 fractions). The most serious late complication after such irradiation is cardiotoxicity. According to Darby et al. the risk of serious cardiac events begins within a few years after the heart has been exposed to ionizing irradiation, which increases linearly with the mean dose to the heart by $7.4 \%$ per gray, with no apparent threshold and continues on for at least 20 years after the irradiation [16]. For this reason, the different and sophisticated methods of irradiation using the deep inspiration breath holding technique should be used to reduce the dose for the heart and coronary vessels, thus diminishing the risk of cardiotoxicity. This is why, special attention should be given to cardiac function during patient follow up. Unfortunately, outcomes for treating breast sarcoma patients have not improved much over the last fifty years. Disease free survival at 5 years in a major series of 63 breast sarcoma patients treated at the Royal Marsden Hospital in London and the Mater Adult Hospital in Australia between 1996-2006 as well as 113 breast sarcomas patients treated at the Warsaw Center for Oncology Institute between 1947-1997 were similar 42\% and $49 \%$ respectively $[17,18]$. Postsurgical irradiation with modern techniques appears to be helpful in such difficult clinical circumstances as in our case, thereby delivering a better chance for curative treatment.

\section{Conclusion}

Synchronous irradiation of both sides of the chest wall after bilateral mastectomy with a single isocenter VMAT technique is possible and well tolerated.

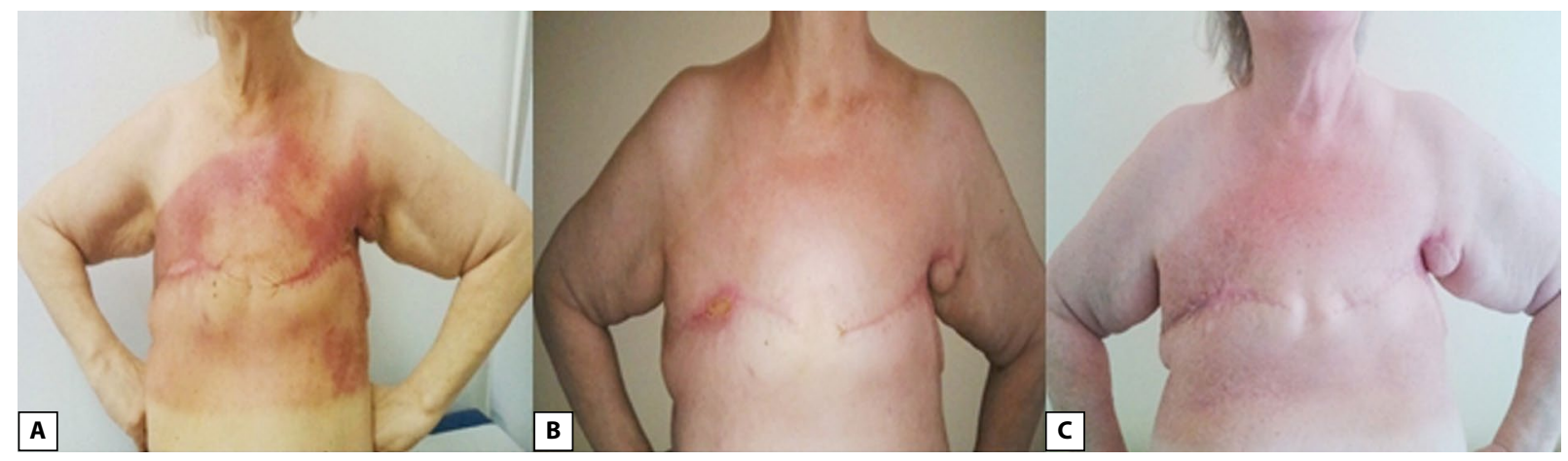

Figure 8. Patient 3 months (A), 6 months (B), one year (C) after irradiation 


\section{Conflict of interest: none declared}

\section{Jacek Gałecki, MD, PhD}

Department of Radiotherapy I

Maria Skłodowska-Curie Memorial Cancer Centre

and Institute of Oncology

\section{Wawelska $15 B$}

02-034 Warszawa, Poland

e-mail:jacek.galecki@coi.pl

Received: 3 Jan 2017

Accepted: 20 Mar 2017

\section{References}

1. Jobsen JJ, van der Palen J, Ong F et al. Synchronous, bilateral breast cancer: prognostic value and incidence. Breast 2003; 12: 83-88.

2. Yamauchi $C$, Mitsumori $M$, Nagata $Y$ et al. Bilateral breast-conserving therapy for bilateral breast cancer: results and consideration of radiation technique. Breast Cancer 2005; 12: 135-139.

3. Grabowski J, Salzstein SL, Sadler GR et al. Malignant phyllodes tumors: a review of 752 cases. Am Surg 2007; 73: 967-969.

4. Cox JD, Stetz J, Pajak TF. Toxicity criteria of the Radiation Therapy Oncology Group (RTOG) and the European Organization for Research and Treatment of Cancer (EORTC). Int J Radiat Oncol Biol Phys 1995; 31: 1341-1346.

5. Hoeller U, Tribius S, Kuhlmey A et al. Increasing the rate of late toxicity by changing the score? A comparison of RTOG/EORTC and LENT/SOMA scores. Int J Radiat Oncol Biol Phys 2003; 55: 1013-1018.

6. Dijkema IM, Hofman P, Raaijmakers CP et al. Loco-regional conformal radiotherapy of the breast: delineation of the regional lymph node clinical target volumes in treatment position. Radiother Oncol 2004; 71: 287-295.

7. Nielsen $\mathrm{MH}$, Berg M, Pedersen AN et al. Delineation of target volumes and organs at risk in adjuvant radiotherapy of early breast cancer: national guidelines and contouring atlas by the Danish Breast Cancer Cooperative Group. Acta Oncol 2013; 52: 703-710.

8. Offersen BV, Boersma $\mathrm{LJ}$, Kirkove $\mathrm{C}$ et al. ESTRO consensus guideline on target volume delineation for elective radiation therapy of early stage breast cancer. Radiother Oncol 2015; 114: 3-10.

9. Feng $M$, Moran JM, Koelling $T$ et al. Development and validation of a heart atlas to study cardiac exposure to radiation following treatment for breast cancer. Int J Radiat Oncol Biol Phys 2011; 79: 10-18.

10. Kong FM, Ritter T, Quint DJ et al. Consideration of dose limits for organs at risk of thoracic radiotherapy: atlas for lung, proximal bronchial tree, esophagus, spinal cord, ribs, and brachial plexus. Int J Radiat Oncol Biol Phys 2011; 81: 1442-1457.

11. Kirkpatrick JP, van der Kogel AJ, Schultheiss TS. Radiation dose-volume effects in the spinal cord. Int J Radiat Oncol Biol Phys 2010; 76 (3 Suppl): S42-S49.

12. Tan W, Liu D, Xue C et al. Anterior myocardial territory may replace the heart as organ at risk in intensity-modulated radiotherapy for left-sided breast cancer. Int J Radiat Oncol Biol Phys 2012; 82: 1689-1697.

13. Depuydt T, Van Esch A, Huysken DP. A quantitative evaluation of IMRT dose distributions: refinement and clinical assessment of the gamma evaluation. Radiother Oncol 2002; 62: 309-319.

14. Seppälä J, Heikkilä J, Myllyoja K et al. Volumetric modulated arc therapy for synchronous bilateral whole breast irradiation - A case study. Rep Pract Oncol Radiother 2015; 20: 398-402.

15. Popescu CC, Olivotto IA, Beckham WA et al. Volumetric modulated arc therapy improves dosimetry and reduces treatment time compared to conventional intensity-modulated radiotherapy for locoregional radiotherapy of left-sided breast cancer and internal mammary nodes. Int J Radiat Oncol Biol Phys 2010; 76: 287-295.

16. Darby SC, Ewertz M, McGale P et al. Risk of ischemic heart disease in women after radiotherapy for breast cancer. N Engl J Med 2013; 368: 987-998.

17. Pencavel $\mathrm{T}$, Allan $\mathrm{CP}$, Thomas JM et al. Treatment for breast sarcoma: A large, single-centre series. Eur J Surg Oncol 2011; 37: 703-708.

18. Niwińska A, Chmielewski R. Fifty years experience in the treatment of breast sarcomas and carcinomas at the Institute of Oncology in Warsaw. An analysis of 113 cases. Nowotwory 2000; 50 (Suppl 2): S54-60. 